\title{
EDITORIAL
}

\section{Clarification of the APJCP Editorial Policy}

\author{
Malcolm Anthony Moore ${ }^{1,2}$, Xinen Huang ${ }^{3}$
}

\author{
Asian Pac J Cancer Prev, 16 (7), 2589-2590
}

It has come to our attention that a number of Chinese authors of papers submitted to the APJCP have expressed concerns and complaints about the policy of the journal regarding criteria for acceptance. As Chief/Deputy Chief Editors we wish to categorically state that it is not the wish of ourselves or any of our colleagues on the Editorial Board that undue preference be given by authors to the APJCP when selecting scientific citations for their reference lists. We simply expect all authors to be scrupulously fair in giving credit to their scientific colleagues in Asia for directly relevant research.

The APJCP is the official journal of the Asian Pacific Organization for Cancer Prevention (APOCP) and as such is committed to supporting its aims - to promote an increased awareness in all areas of cancer prevention/ control and to stimulate research and practical intervention approaches in the Asian Pacific area. We therefore give most emphasis to research work which hopefully will contribute to practical cancer control measures in the countries of the region. Most scientists and other interested parties feel that there are major differences in the problems facing Asia from those in the developed world and the APJCP was founded to cater primarily to a developing world audience.

Given the daunting difficulties faced by many Asian authors trying to publish in the established cancer journals in North America and Europe the APJCP was launched to provide a more accessible forum to our colleagues in the region. It never aspired, and does not aspire, to be a cutting edge journal competing with the high impact journals of the Western world, which have their own clientele. The stress is on epidemiology and population-based research but naturally we are also aware of the necessity for basic science and clinical studies pertinent to our populations.

Since there are often financial and other resource limitations to the type of research which can be funded in the Asian setting, we set particular store on the quality of submitted manuscripts in the one area where there is a level playing field - that of the reference list. Given the free access to PubMed, all scientists, independent of their physical location, can provide a thorough coverage of the relevant literature. The only feasible method that we have at our disposal to assess the quality of the referencing of articles submitted to the APJCP is to ascertain whether the papers which are directly appropriate and have appeared in its pages are included. If not, we can only conclude that the coverage is not comprehensive or that a negative bias may be present. Either of these are grounds for careful consideration on the part of any responsible Editorial Board.

I repeat, we urge all authors not only to cite the papers which have a direct bearing on their own work in the APJCP but also those in all other journals, especially those examples published in Asia. It should naturally be stressed that we do not have any limitation on the number of citations that can be included in any category of publication in the APJCP.

With the support of the APOCP and backing from the National Cancer Center in Korea and the UICC Asian Regional Office the aim has always been to provide a relatively inexpensive journal (basically our formatting fees cover only the costs of our offices in Bangkok, Thailand, and Nanjing, China), featuring rapid processing and timely publication, open access, and an Impact Factor commensurate with its contribution to the world and especially Asian literature on cancer control research. We are well aware that we have not always been successful in this endeavour (Moore et al., 2014), but we have managed to publish over 1,800 papers in 2014 (more than 450 so far in six issues in 2015), and will continue to do our utmost to provide an efficient service.

A brief comparison of the number of articles in the International Journal of Cancer, published by the UICC, and in the APJCP, with support of the UICC-ARO, is revealing in terms of the relative weight given to different countries (see Table 1). We leave the reader to draw his or her own conclusions. While we hoped that we might be able to also increase our Impact Factor by focusing on awareness (Moore et al., 2013), the fact that we did not achieve our 'aim' is concrete evidence that editorial policy in practice was not to artificially exert an influence by limiting the number and type of accepted papers. Rather we continued our commitment to the scientists in the region with whom we have a reciprocal dependence.

To return to the issue raised in the first paragraph of this editorial, we would be happy to receive comments and criticisms of the APJCP policy from any country. Our automatic rejection of any paper with less than $20 \%$ of 
Table 1. Comparison of Country of Origin in PubMed Listed Articles, 2014

\begin{tabular}{lrr}
\hline & Int J Cancer & APJCP \\
\hline Australia & 31 & 10 \\
Bangladesh & 0 & 4 \\
Brunei & 0 & 4 \\
China & 141 & 790 \\
Egypt & 0 & 19 \\
France & 57 & 4 \\
Germany & 63 & 0 \\
India & 7 & 119 \\
Indonesia & 1 & 12 \\
Iran & 4 & 185 \\
Japan & 66 & 48 \\
Jordan & 0 & 6 \\
Kazakhstan & 0 & 3 \\
Korea & 30 & 67 \\
Malaysia & 2 & 73 \\
Mongolia & 1 & 4 \\
Nepal & 0 & 9 \\
Oman & 0 & 2 \\
Pakistan & 1 & 44 \\
Saudi Arabia & 0 & 8 \\
Thailand & 2 & 237 \\
Turkey & 1 & 16 \\
United States & 214 & 3 \\
United Kingdom & 93 & 3 \\
Vietnam & 0 &
\end{tabular}

citations is based on the fact that we wish to stress research areas which are of continuing interest. We consider it self evident that this is an important point and will look at any resubmission in which the literature is brought up -to -date without any bias. Similarly, submissions which have not been prepared in line with our Instructions to Authors will be sent back with a request that the formatting is appropriate and also that the relevant referencing be as comprehensive as possible.

We trust that authors considering submission will understand this policy, set by the APOCP and our Editorial Board with representatives across the Asian Pacific. For our part, we also understand the career-driven agenda of many scientists and wish them success in publishing in the Western world. As always we would like to express our appreciation to all those who choose the APJCP for publication of their research findings, with a plea for exhaustive discussion of their research implications in light of the previously published literature, whether in our journal or elsewhere.

\section{References}

Moore MA, Lim MK, Huang X (2014). Apologies for past mistakes and promises for a new start for the APJCP in 2014. Asian Pac J Cancer Prev, 15, 1-2.

Moore MA, Lim MK, Huang X (2013). New APJCP impact factor for 2012 is 1.271--time to make a major effort to break through the 2.0 barrier. Asian Pac J Cancer Prev, 14, 3377-8. 